\title{
ESTILOS DE LIDERANÇA E DESEMPENHO DE EQUIPES NO SETOR PÚBLICO
}

\author{
Maria Aparecida Muniz Jorge Dias* \\ mariamuniz2@yahoo.com.br \\ Renata Simões Guimarães e Borges* \\ renatasg@face.ufmg.br \\ *Universidade Federal de Minas Gerais - Belo Horizonte, MG
}

http://dx.doi.org/10.1590/1413-2311.0542014.53468

Recebido em 09/10//2014

Aprovado em 11/03/2015

Disponibilizado em 01/04/2015

Avaliado pelo sistema double blind review

Revista Eletrônica de Administração

Editor: Luís Felipe Nascimento

ISSN 1413-2311 (versão on line)

Editada pela Escola de Administração da Universidade Federal do Rio Grande do Sul.

Periodicidade: Quadrimestral

Sistema requerido: Adobe Acrobat Reader.

\section{RESUMO}

O presente trabalho tem por objetivo analisar como os estilos de liderança dos gestores públicos influenciam o desempenho das equipes. Com base em pesquisas anteriores, a hipótese colocada neste estudo é que o estilo de liderança transformacional levaria aos melhores resultados de desempenho. Com isso, foi realizado um survey no poder executivo do estado de Minas Gerais, cuja amostra obtida foi de 315 servidores representando 82 equipes. Questionários padronizados e baseados em instrumentos amplamente testados foram aplicados. Os resultados desta pesquisa não confirmam a literatura sobre liderança, pois os servidores apontam que o desempenho das equipes é influenciado positivamente pelo estilo de liderança transacional e não transformacional. Ou seja, os líderes que utilizam mecanismos de negociação que atrelam o desempenho a recompensa são os que obtêm melhores resultados. Esta pesquisa revela que na administração pública o estilo de liderança transacional está associado ao melhor desempenho das equipes, cujas características possuem uma interface com o novo modelo da política de gestão de pessoas, que tem como um de seus pilares a meritocracia. No entanto, é preciso repensar o papel da liderança no setor público uma vez que a liderança transacional excessiva leva a um baixo envolvimento do funcionário. Este aspecto é evidenciado na medida de desempenho que revela que o comprometimento profissional das equipes é o mais baixo índice de desempenho.

Palavras-Chave: Liderança; Estilos de Liderança; Desempenho de Equipes; Gestão de Pessoal; Administração Pública.

\section{LEADERSHIP STYLES AND TEAM PERFORMANCE IN PUBLIC SECTOR}

\footnotetext{
ABSTRACT

The aim of this paper is to evaluate how leadership styles influence team performance in public administration. Based on previous research, the hypothesis of this study posits that the

REAd | Porto Alegre - Edição 80 - º 1 - janeiro/abril 2015 - p. 200-221
} 
transformational leadership style would lead to better results on performance. With that, a survey was employed in the executive branch of Minas Gerais state, in which a sample size of 315 public employees representing 82 work teams was obtained. We applied standardized questionnaires based on well tested instruments available in the literature. The results of this research do not support the literature on leadership because public employees suggest that team performance is influenced positively by the transactional leadership style instead of transformational leadership style. In other words, leaders who employ negotiation mechanism to link performance and reward are those who obtain better results. This research reveals that in public administration the transactional leadership style is more associated with better team performance, which embodies the new model of personnel management politics that has one of its pillars represented by meritocracy. Nevertheless, it is necessary to rethink the role of leaders in public sector because transactional leadership style in excess leads to a low employee involvement. This aspect is illustrated at the performance measurement of professional commitment, which is the lowest index of performance.

Keywords: Leadership; Leadership Styles; Team Performance; HR Management; Public Management.

\title{
ESTILOS DE LIDERAZGO Y DESEMPEÑO DEL EQUIPO EN EL SECTOR PÚBLICO
}

\begin{abstract}
RESUMEN
Este estudio tiene como objetivo examinar cómo los estilos de liderazgo de los gestores públicos influyen en el rendimiento del equipo. Basado en la investigación anterior, la hipótesis planteada en este estudio es que el estilo de liderazgo transformacional conduciría a mejores resultados en el rendimiento. Con eso, se realizó una encuesta en la rama ejecutiva del estado de Minas Gerais, cuya muestra incluyó 82 equipos que representan a 315 empleados públicos. Se aplicaron cuestionarios estandarizados basados en instrumentos ya probados disponibles en la literatura. Los resultados de esta investigación no son compatibles con la literatura sobre el liderazgo porque los empleados públicos sugieren que el rendimiento del equipo se ve influenciado positivamente por el estilo de liderazgo transaccional en lugar del estilo de liderazgo transformacional. En otras palabras, los líderes que emplean mecanismo de negociación que vinculan el rendimiento y la recompensa son los que obtienen mejores resultados. Esta investigación revela que en la administración pública el estilo de liderazgo transaccional se asocia más con un mejor rendimiento del equipo, que incorpora el nuevo modelo de la política de gestión de personal que tiene uno de sus pilares representados por la meritocracia. Sin embargo, es necesario repensar el papel de los líderes en el sector público porque el liderazgo transaccional en exceso conduce a una baja participación de los empleados. Este aspecto se ilustra en la medición del rendimiento de compromiso profesional, que es el índice más bajo de rendimiento.
\end{abstract}

Palavras Clave: Liderazgo; Estilos de Liderazgo; Rendimiento del Equipo; Gestión de Recursos Humanos; Gestión Pública.

\section{INTRODUÇÃO}

Nos últimos anos, as organizações vêm realizando grandes transformações nas práticas de gestão e na maneira como organizam o trabalho, refletindo em mudanças significativas REAd | Porto Alegre - Edição 80 - º 1 - janeiro/abril 2015 - p. 200-221 
Estilos de liderança e desempenho de equipes no setor público

percebidas nas práticas culturais e político-econômicas que vêm ocorrendo no mundo, a partir do início da década de 1970, e posteriormente, no Brasil. Essas transformações fizeram com que as organizações substituíssem um modelo baseado na hierarquia, na racionalização e divisão do trabalho cuja ênfase estava nas rotinas, no controle e na disciplina, por outro mais flexível voltado para a multifuncionalidade, à mudança e à inovação (HARVEY, 1999).

Nesse contexto, a administração pública do Poder Executivo de Minas Gerais, há 10 anos, vem passando por diversas mudanças a partir da implementação do Programa Choque de Gestão, que já se encontra em sua terceira geração. Um conjunto de medidas voltado para a modernização da gestão pública foi estruturado e implantado, tendo as lideranças como os principais impulsionadores desse processo. De acordo com Abrucio (2007), esses atores são fundamentais para a viabilização das estratégias governamentais concebidas em um novo modelo de gestão pública, guiado pela inovação, pelo dinamismo e capaz de estabelecer um novo paradigma administrativo, com foco sobre a entrega de resultados para os cidadãos.

Somado a isso, tem-se que um processo de gestão de desempenho efetivo, sob um enfoque sistêmico, depende não só de instrumentos e estratégias, mas, sobretudo, de uma liderança eficaz e eficiente (SOUZA et al., 2005). O desenvolvimento de equipes eficazes depende da liderança, pois, ao confiarem nos líderes, os subordinados ficam dispostos a assumir riscos para alcançar os objetivos (SHEN; CHEN, 2007). Os membros da equipe acreditam que seus direitos e interesses não serão desconsiderados pelo líder. Assim, há um efeito positivo entre liderança e compromisso do liderado. Corroborando com esse entendimento, Benedetti et al. (2004) afirmam que o desempenho diferenciado é alcançado a partir da criação de uma visão estimulante de futuro por parte dos líderes.

Além da liderança, a gestão de desempenho tornou-se fundamental para a reforma da gestão pública no início do século XXI (MOYNIHAN, 2008). A mudança de atenção de regras e regulamentos para o estabelecimento de metas e a utilização de informações sobre o desempenho tem sido visto como uma tentativa de melhorar o desempenho do setor público (HOOD, 1991; FERLIE et al., 1996; POLLITT; BOUCKAERT, 2004). Segundo De Waal (2010), estudos no setor público mostraram que através da implementação da gestão do desempenho, as organizações públicas têm mais chances para alcançar seus objetivos, oferecer melhores serviços aos cidadãos e empresas, e melhorar a sua eficiência global.

Por estas razões, forte ênfase é colocada na promoção do trabalho em equipe e da liderança forte (JONES; RUDD, 2007). Assim, o grande desafio dessas lideranças é fazer com que o potencial das equipes seja direcionado ao alcance dos objetivos e resultados 
organizacionais. As equipes são uma unidade básica de desempenho, sendo necessário mobilizá-las para que trabalhem efetivamente em equipe e, assim, consigam gerar os resultados almejados. Portanto, o papel dos líderes no contexto atual da administração pública assume é destacado devido à necessidade cada vez mais premente de melhoria do desempenho organizacional, o que exige evidentemente a melhoria do desempenho das equipes.

Com isso, o presente trabalho tem por objetivo analisar como os estilos de liderança dos gestores públicos influenciam o desempenho das equipes da administração direta, autárquica e fundacional do Poder Executivo do Estado de Minas Gerais. Especificamente, esta pesquisa pretende compreender qual estilo de liderança - transformacional, transacional ou laissez-faire - está mais associado com o bom desempenho das equipes.

\section{REFERENCIAL TEÓRICO}

\subsection{Liderança organizacional}

No campo da gestão e do comportamento organizacional, o estudo da liderança tem sido uma parte importante e central da literatura há várias décadas (YUKL, 1989). São inúmeros os significados que o termo liderança assume, dependendo da área de estudo que o utiliza. Em geral, a liderança é entendida como um processo de influência das atividades de um grupo organizado em seus esforços para o estabelecimento de metas e realização de objetivos (STOGDILL, 1950; RAUCH; BEHLING, 1984). Katz e Kahn (1978) definem a liderança como a influência incremental sobre o cumprimento mecânico com as diretrizes rotineiras da organização.

No entanto, para outros autores a liderança supera a influência mecanicista e incremental para dar sentido e propósito ao esforço coletivo (JACOBS; JAQUES, 1990; YUKL, 1994), trabalhando principalmente a influência interpessoal através da comunicação (TANNENBAUM et al., 1961; SADLER, 2003). Neste sentido, Bass (1990) aborda a liderança como centro no processo de grupo, como uma questão de personalidade, como um exercício de influência e de persuasão, como resultante de comportamentos específicos, como uma relação de poder, como um instrumento para alcançar metas, como um esforço de interação, como um papel diferenciado, como a iniciação da estrutura e como muitas combinações dessas definições.

Para Bergamini (1994), dois aspectos parecem ser comuns à grande maioria das definições de liderança existentes na atualidade. Primeiramente, elas apresentam o 
Estilos de liderança e desempenho de equipes no setor público

denominador comum de que a liderança esteja ligada a um fenômeno grupal, ou seja, envolve duas ou mais pessoas. Pois, a liderança não ocorre no isolamento, ou seja, não há líderes sem seguidores (DORFMAN, 1996). Em segundo lugar, evidenciam um processo de influencia exercido de forma intencional por parte dos líderes sobre seus liderados. Para esta autora, Hollander (1978) consegue abranger a grande maioria desses aspectos propondo que o processo de liderança geralmente envolve um relacionamento de influência em duplo sentido, orientado principalmente para o atendimento de objetivos mútuos, tais como aqueles de um grupo, organização ou sociedade. Portanto, a liderança não é apenas o cargo do líder, mas também requer esforços de cooperação por parte de outras pessoas.

Atualmente, os esforços têm se concentrado nas abordagens da liderança transacional e da liderança transformacional. Burns (1978) foi um dos primeiros a fornecer uma definição explícita de liderança transformacional. Ele propôs que o processo de liderança ocorre em uma das duas maneiras, transacional ou transformacional. A liderança transacional é baseada na autoridade burocrática e legitimidade dentro da organização. Líderes transacionais enfatizam padrões de trabalho, atribuições e tarefas orientadas para os objetivos. Eles também tendem a se concentrar na conclusão da tarefa e na disciplina do funcionário e confiam demais em recompensas organizacionais e punições para influenciar o desempenho. Em contraste, Burns (1978) caracteriza a liderança transformacional como um processo que motiva seguidores apelando aos ideais mais elevados e aos valores morais. Os líderes transformacionais devem ser capazes de definir e articular uma visão para suas organizações, e os seguidores devem aceitar a credibilidade do líder.

Posteriormente, Bass e Avolio (1994) desenvolveram uma teoria da liderança transformacional que é um ponto culminante e de extensão do trabalho anterior de Bennis e Nanus (1985), Burns (1978), Tichy e Cohen (1997) e outros. Bass e Avolio (1997) propuseram três estilos de liderança - liderança transacional, liderança transformacional e laissez-faire.

$\mathrm{Na}$ liderança transacional, o comportamento do líder é direcionado para o acompanhamento e orientação do subordinado, distribuição de recompensas, no papel de educador e motivador da equipe e no relacionamento com as pessoas, visando atingir as metas e objetivos organizacionais (NORD; FOX, 1996). O foco principal de líderes transacionais é nos objetivos, esclarecendo a ligação entre desempenho e recompensas, bem como no feedback construtivo para manter os seguidores na tarefa (BASS, 1985; JUNG; AVOBIO, 1999). No caso da liderança transacional, de acordo com Bass (1985), os comportamentos

REAd | Porto Alegre - Edição 80 - N 1 - janeiro/abril 2015 - p. 200-221 
típicos são: recompensa contingente, administração por exceção ativa, administração por exceção passiva. A recompensa contingente refere-se a uma troca de recompensas entre líderes e seguidores, ou seja, são oferecidas recompensas para o bom desempenho ou feitas ameaças e disciplinas para desempenhos ruins. O líder que depende fortemente da administração por exceção passiva intervém com o seu grupo apenas quando procedimentos e normas para a realização de tarefas não são cumpridas. Em contraste, na administração por exceção ativa, os líderes são caracterizados como monitores que detectam erros (BASS, 1985).

Já a liderança transformacional é definida como um processo em que um líder tenta aumentar a sensibilidade dos seguidores acerca do que era certo e importante, para motivá-los a realizar além das expectativas (BASS, 1985). Bass e Avolio (1997) indicaram que os líderes transformacionais normalmente apresentam comportamentos associados a quatro características:

- Influência idealizada: ocorre quando o líder é um modelo para os seus seguidores, incentivando-os a compartilhar visões e objetivos comuns e fornecendo uma visão clara e um forte senso de propósito;

- Motivação inspiradora: representa comportamentos em que um líder tenta expressar a importância dos objetivos desejados de maneira simples, comunica o elevado nível de expectativas e proporciona aos seguidores significado e desafios com o trabalho;

- Estímulo intelectual: refere-se aos líderes que desafiam as ideias dos seguidores e valores para a solução de problemas;

- Consideração individualizada: refere-se a líderes que passam mais tempo ensinando e treinando seguidores, tratando-os de forma individualizada.

Segundo Neto et al. (2012), além dessas características, os líderes transformacionais consideram-se agentes de mudança, pois são designados para transformar a organização a que pertencem. Têm condições de lidar com a resistência, tomar uma posição, assumir riscos e confrontar a realidade. Enxergam o erro como uma possibilidade de aprendizado e podem enfrentar a complexidade e a incerteza. O líder transformacional busca ir além do instrumentalismo transacional.

Por fim, o estilo de liderança laissez-faire caracteriza-se pelo fato do líder interferir pouco nas decisões do grupo, ou seja, ele dá liberdade para que os colaboradores tomem as REAd | Porto Alegre - Edição 80 - N 1 - janeiro/abril 2015 - p. 200-221 
Estilos de liderança e desempenho de equipes no setor público

decisões. Nesse estilo, o líder evita esclarecer suas expectativas e resolver os conflitos, bem como transfere sua autoridade para os liderados, abdicando deliberadamente do poder de tomar decisões, que são delegadas aos liderados (BASS; AVOLIO, 2000).

\subsection{Desempenho de equipes e estilos de liderança}

No setor público, de acordo com Dooren et al. (2010), o termo desempenho acumula significados múltiplos e, muitas vezes, ambíguos. No entanto, para esse autor, é possível inferir um conceito universal para desempenho, traduzindo-o como um comportamento intencional que pode ser individual ou organizacional. A partir dessa definição de desempenho como ação deliberada, Dooren et al. (2010) constroem uma classificação baseada em quatro perspectivas: desempenho como produtividade; desempenho como competências/capacidade, desempenho como bons resultados e desempenho como resultados sustentáveis. A primeira perspectiva de desempenho concentra a atenção em todas as ações que são realizadas e no comportamento intencional dos atores governamentais. Essa concepção é de natureza relativamente imparcial, mas também é muito ampla.

As outras perspectivas do conceito de desempenho contêm um juízo de valor. O desempenho tem uma qualidade que pode ser elevada ou baixa. Primeiramente, quando o desempenho é sobre a qualidade das ações, e não tanto sobre a qualidade dos resultados, ele é conceituado como competência ou capacidade. Em segundo lugar, quando o desempenho é sobre a qualidade dos resultados e não tanto sobre a qualidade das ações, o desempenho é igual a resultados (a capacidade da organização não é o foco desta conceituação). A Nova Gestão Pública vê o desempenho sob essa última perspectiva. Enquanto os resultados são comprovados, não importa como eles surgiram. Finalmente, quando o desempenho é conceituado considerando tanto a qualidade das ações quanto a qualidade dos resultados, pode ser tipificado como resultados sustentáveis. Desempenho refere-se à organização produtiva, ou seja, uma organização que tem a capacidade de realizar e converter essa capacidade em resultados (DOOREN et al., 2010).

Existem diferentes modelos de mensuração de desempenho, que envolvem três níveis de análise: organizacional, de equipe ou individual (BRANNICK; PRINCE, 2009). Um sistema de gestão de desempenho em diferentes níveis da organização é descrito por Guimarães et al. (1998). No nível corporativo, o planejamento, o acompanhamento e a avaliação restringem-se à missão, visão e objetivo macro, tendo a sustentabilidade da 
organização como resultado esperado. No nível divisional ou funcional, o interesse recai sobre os objetivos e metas de cada unidade produtiva da empresa, visando à eficácia organizacional. No nível grupal, a avaliação concentra-se sobre os projetos e processos de trabalho, isto é, sobre as equipes. E, finalmente, no nível individual, o objeto a ser avaliado é o resultado do trabalho do indivíduo, do seu comportamento no ambiente de trabalho, ou de ambos. Nestes dois últimos níveis, busca-se a qualidade e produtividade dos produtos e serviços gerados pela organização. As organizações têm buscado trabalhar com a medição do desempenho nestes diferentes níveis, de acordo com o grau de maturidade no que se refere ao planejamento e a utilização de ferramentas de mensuração de resultados.

Considerando o âmbito de atuação de uma liderança, é possível avaliar a influência do seu estilo tanto em nível individual, quanto em nível de equipe. Apesar da unidade tradicional de avaliação em uma organização ser o indivíduo, na pesquisa realizada foi mensurado apenas o nível de desempenho de equipes, pois cada vez mais o foco está na equipe para o cumprimento de metas e os indivíduos são dependentes de outros com quem trabalham. Assim, o desempenho de qualidade requer um esforço de equipe, e o sucesso, em grande medida, depende da capacidade de um indivíduo para gerir as pessoas, bem como os recursos materiais (MARGERISON et al., 1995).

Murphy (2008) considera que a melhor forma de utilização da avaliação de desempenho é como parte de um conjunto de medidas convergentes que, aplicadas juntamente, apresenta maior chance de captar a variação no desempenho do trabalho do que se usasse apenas uma medida isoladamente. Nesse sentido, Fernandes (2013) sugere um modelo integrado de avaliação de desempenho que capte diversas dimensões. Existem avaliações de entradas e saídas. As entradas são avaliadas com base nas capacidades, que podem ser conhecimentos, habilidades, atitudes e valores. Conhecimentos e habilidades dispensam sistemática de avaliação; são registrados nos currículos dos profissionais, em termos de treinamentos e formação acumulados, e, quando muito, validados por meio de certificados. As saídas são avaliadas em termos de entregas estabelecidas nas competências e os resultados aferidos por indicadores dentro de um sistema de metas. As entregas são analisadas por auto-avaliação e avaliação dos superiores, ao passo que os resultados são avaliados em termos de indicadores e, em seguida, armazenados e disponibilizados em sistemas operacionais.

As pesquisas realizadas por Wayne et al. (1999) e Waldman et al. (2001) indicam que a liderança eficaz pode melhorar o desempenho dos indivíduos e das organizações, não sendo

REAd | Porto Alegre - Edição 80 - º 1 - janeiro/abril 2015 - p. 200-221 
Estilos de liderança e desempenho de equipes no setor público

estudado os benefícios da liderança também para as equipes. Uma razão pela qual o desempenho das equipes pode diferir (do individual e organizacional) é a potencial incapacidade de um líder para criar uma visão global, que é um componente crítico de liderança organizacional eficaz (BASS et al., 2003). Dadas estas potenciais diferenças, uma revisão quantitativa dos efeitos de liderança no nível de desempenho da equipe parece especialmente justificada (STEWART, 2006).

A literatura que procura identificar como os estilos de liderança afetam os resultados organizacionais tem focado na liderança transformacional como a mais eficaz. Ross e Offermann (1997) examinaram os efeitos dos atributos de personalidade dos líderes transformacionais sobre a satisfação e o desempenho dos subordinados. O estudo identificou que embora o estilo de liderança transformacional tenha se correlacionado significativamente com a satisfação dos subordinados, esse tipo de liderança não apresentou correlações significativas com os critérios de desempenho adotados na pesquisa.

Jung e Avolio (1999) pesquisaram os estilos de liderança transformacional e transacional e compararam-nos em condições de tarefas individuais e de grupo para determinar se eles tinham diferentes impactos sobre o desempenho individual e coletivo. Realizando uma tarefa de brainstorming, os resultados mostraram que os grupos com um líder transformacional geraram mais ideias, mas, individualmente geraram mais ideias com um líder transacional. No geral, o desempenho do grupo foi mais elevado do que a dos indivíduos que trabalham isoladamente. No entanto, ao contrário das expectativas, os grupos geraram mais ideias que exigiam mudanças organizacionais fundamentais no trabalho individual.

Dionne et al. (2004) construíram um modelo que possibilita testar se as dimensões específicas de liderança transformacional (influência idealizada, motivação inspiradora, consideração individualizada e estímulo intelectual) pode produzir resultados intermediários que poderiam gerar um impacto positivo sobre os processos interpessoais da equipe, e, como tal, melhoraria o desempenho da mesma. Segundo estes autores, comportamentos transformacionais podem impactar processos subjacentes do trabalho em equipe como comunicação, gestão de conflitos e coesão.

Por sua vez, Moynihan et al. (2012) pesquisaram sobre a influência da liderança transformacional na percepção de burocracia por parte dos funcionários. Os resultados mostraram que os comportamentos de liderança transformacional podem alterar as percepções da burocracia através de seus efeitos sobre três principais variáveis mediadoras - clareza das metas organizacionais, comunicação interna, e apoio político. E essas relações de mediação se 
mantêm até mesmo quando controladas as variáveis intervenientes - o tamanho da agência e a função (prestação de serviço externo versus controle). Os achados deste estudo ressaltaram que é fácil perder os efeitos indiretos da liderança. Os líderes podem fazer a diferença em uma questão específica organizacional, se quiserem. Mas, os líderes também podem ter impacto de longo alcance e imprevisto na vida organizacional.

Examinando o impacto da experiência subjetiva do poder na dinâmica da liderança e do desempenho da equipe, Tost et al. (2013) verificaram que o efeito psicológico do poder em líderes formais afeta o desempenho da equipe. Argumenta-se que a elevada experiência de poder de um líder formal produz o domínio verbal, o que reduz a comunicação da equipe e, consequentemente, diminui o desempenho. Importante, porque estas dinâmicas contam com a aquiescência de outros membros da equipe para o comportamento dominante do líder, os efeitos só surgem quando o líder tem uma posição de liderança formal.

Em suma, os achados das pesquisas sugerem que a liderança exerce um papel fundamental, tendo em vista que sua atuação reflete na satisfação; na geração de ideias e reflexão da equipe que, por sua vez, promove a inovação. Além disso, pode impactar na comunicação, gestão de conflitos, coesão e confiança da equipe. Tudo isso, consequentemente, gerará efeitos no próprio desempenho da equipe, sendo importante analisar a dinâmica da díade líder-liderado de forma a identificar o estilo de liderança mais eficaz para melhorar esse desempenho. Em relação ao estilo de liderança, os estudos têm apontado que o estilo transformacional exerce uma maior influência no desempenho individual e no desempenho de equipes.

Portanto, a seguinte hipótese de pesquisa é colocada:

H1. O estilo de liderança transformacional influenciará mais o desempenho de equipes quando comparado com os estilos de liderança transacional e laissez-faire.

\section{METODOLOGIA}

Esta pesquisa é de natureza explicativa, pois busca estabelecer correlações entre variáveis, definindo sua natureza e servindo de base para explicar os fenômenos que descreve (GIL, 2006). Utilizou-se o método survey para a coleta de dados, pois será utilizada uma amostra para fazer inferências sobre atitudes, comportamentos e características de uma população que, de tão grande, inviabiliza-se sua observação direta (CRESWELL, 2007). O

REAd | Porto Alegre - Edição 80 - º 1 - janeiro/abril 2015 - p. 200-221 
Estilos de liderança e desempenho de equipes no setor público

survey permite a utilização de testes rigorosos para verificar proposições empíricas relativas ao relacionamento de diversas variáveis e examinar cuidadosamente a importância relativa de cada uma delas (BABBIE, 1999).

Portanto, a abordagem da pesquisa é quantitativa com a finalidade de identificar os estilos de liderança presentes na administração direta, autárquica e fundacional e explicar as possíveis relações com o desempenho das equipes, que também é mensurado em termos quantitativos. Segundo Collins e Hussey (2005), o estudo quantitativo se caracteriza por transformar opiniões e informações em números que possibilitam a classificação e análise dos dados coletados nos instrumentos aplicados.

A população da pesquisa é constituída pelos ocupantes de cargos de direção e de chefia das estruturas básica e intermediária, exceto a alta direção, dos 58 órgãos e entidades da administração direta, autárquica e fundacional do Poder Executivo do Estado de Minas Gerais, que são submetidos à Avaliação de Desempenho dos Gestores Públicos. Esse critério de corte deve-se ao fato de o perfil de competências gerenciais, que é utilizado para realização dessa avaliação conter a Liderança de Equipes como uma competência requerida, ou seja, espera-se que todos os gestores apresentem as características de um líder e não fiquem limitados às funções gerenciais. Cabe destacar que, nas Secretarias de Estado, a alta direção corresponde aos cargos de Secretário, Secretário-Adjunto e Subsecretários e, nas autarquias e fundações, corresponde aos cargos de Diretor Geral, Vice Diretor Geral, Presidente e Vice Presidente, Reitor e Vice Reitor. A Avaliação de Desempenho dos Gestores Públicos é regulamentada pelo Decreto ${ }^{\circ}$ 44.986, de 19 de dezembro de 2008, sendo realizada com base no perfil de competências gerenciais que compreende: orientação para resultados, visão sistêmica, gestão de pessoas, liderança de equipes, comportamento inovador, compartilhamento de informações e de conhecimentos e competência técnica.

Para realização da pesquisa, o cálculo da amostra foi realizado com base no processo de amostragem aleatória, a fim de garantir que as equipes selecionadas representem adequadamente a população de líderes pesquisados. Para definição da amostra, foi utilizada a análise de poder (power analysis), um teste que busca minimizar o erro estatístico $(\alpha)$, relacionando diretamente o tamanho da amostra e o tamanho do efeito esperado. Dessa forma, utilizando-se o software GPower, versão 1.3.5, atribuindo-se um power de 80\%, para um efeito esperado de 0,15 , um erro de $5 \%$ e 3 preditores (ou seja, três estilos de liderança) o tamanho da amostra da pesquisa precisaria ser de, no mínimo, 77 equipes.

REAd | Porto Alegre - Edição 80 - º 1 - janeiro/abril 2015 - p. 200-221 
Os questionários foram enviados via e-mails a 1750 membros de 350 equipes, explicando o objetivo da pesquisa e disponibilizando o link do Google Docs para acesso ao questionário. O questionário foi respondido por 315 indivíduos sendo 233 liderados e 82 líderes de equipe, representando uma amostra de 82 equipes, superior ao mínimo necessário.

\subsection{Instrumento de pesquisa e análise dos dados}

Para a coleta de dados foi estruturado um questionário composto de três partes: $\mathrm{Na}$ primeira parte, para identificação dos estilos de liderança, foram utilizadas 21 perguntas do Questionário de Liderança Multifator (MLQ), desenvolvido por Bass e Avolio (2000). O MLQ contém 21 itens que medem a gama de comportamentos de liderança, tem sido repetidamente validado por especialistas em liderança e é fortemente preditivo do desempenho do líder (BASS, 1990). Este questionário define medidas de estilos de liderança e comportamentos que variam da liderança transacional para a liderança transformacional, incluindo a liderança laissez-faire. De acordo com Bass e Avolio (2000), a confiabilidade do MLQ para cada liderança fator varia de 0,74-0,91. É o instrumento mais utilizado para avaliar estilos de liderança (KIRKBRIDE, 2006) e é considerada a melhor medida validada de liderança transformacional e transacional (ÖZARALLI, 2003). A escala utilizada na Parte 1 dos questionários é a existente no próprio MLQ, com cinco itens: nunca, correspondente a 0 ponto; raramente, correspondente a 1 ponto; às vezes, correspondente a 2 pontos; frequentemente, correspondente a 3 pontos; e sempre, correspondente a 4 pontos.

A parte 2, composta de 24 perguntas, foi construída a partir das entregas esperadas dos servidores dos órgãos e entidades do Poder Executivo do Estado de Minas Gerais, estabelecido pela Resolução SEPLAG nº 001, de 3 de janeiro de 2013. Essas entregas são agrupadas em cinco (5) blocos referentes aos seguintes temas: foco em resultados, foco no cliente, inovação, trabalho em equipe e comprometimento profissional. As descrições das entregas foram adaptadas, pois para a pesquisa essas foram utilizadas como uma das dimensões da variável desempenho das equipes, conforme modelo proposto por Fernandes (2013). Na parte 2 do questionário, a escala utilizada foi a do tipo Likert de cinco itens: discordo totalmente, correspondente a 1 ponto; discordo, correspondente a 2 pontos; nem concordo nem discordo, correspondente a 3 pontos; concordo, correspondente a 4 pontos; e concordo totalmente, correspondente a 5 pontos.

Finalmente, a última parte era composta por questões relativas aos dados demográficos dos respondentes. Na primeira página do questionário uma carta de apresentação informava os 
Estilos de liderança e desempenho de equipes no setor público

objetivos da pesquisa, garantindo o anonimato do respondente bem como a participação voluntária no estudo.

Realizou-se, primeiramente, a análise descritiva dos dados. Em seguida, foi empregada a análise de correlação, por meio do coeficiente de correlação de Pearson que é uma medida do grau de relação linear entre duas variáveis quantitativas. Finalmente, foi utilizada a análise de regressão múltipla que é um método estatístico de previsão de valores de uma ou mais variáveis de resposta (dependentes) por meio de um conjunto de variáveis explicativas (independentes). No caso da regressão múltipla, também foi analisado o coeficiente de determinação $\left(\mathrm{R}^{2}\right)$ que é o quociente entre a variação explicada e a variação total para todas as variáveis independentes. Este coeficiente pode assumir valores entre 0 e 1, sendo o valor $1 \mathrm{o}$ que representa a situação onde toda a variação é explicada (ANDERSON et al., 2011). Os dados da pesquisa foram tratados com o auxílio do software Statistical Package for the Social Sciences - SPSS.

\section{RESULTADOS}

Analisando-se as variáveis que medem os estilos de liderança, os participantes da pesquisa não percebem uma grande diferenciação entre os estilos de liderança transformacional e o transacional, que apresentam médias muito semelhantes $(M=7,12, D . P$. $=3,40$ e $M=6,96, D . P .=2,70$, respectivamente). No estilo de liderança transformacional, os fatores que mais se destacam são a motivação inspiradora e a influência idealizada $(M=7,39$, D.P. $=3,42$ e $M=7,26, D . P .=3,57$, respectivamente). No estilo de liderança transacional, o fator administração pela exceção é o que apresenta a maior média $(M=7,75, D . P .=2,52)$. Já o estilo de liderança laissez-faire possui a menor média $(M=5,85, D . P .=2,46)$, sendo considerado o menos presente nos líderes dos liderados que responderam à pesquisa. No entanto, pela escala do MLQ, todos os estilos de liderança e seus fatores estão no nível moderado que vai de 5 a 8 pontos, considerando-se as médias. A Tabela 1 apresenta os resultados referentes aos estilos de liderança e seus fatores.

REAd | Porto Alegre - Edição 80 - º 1 - janeiro/abril 2015 - p. 200-221 
Maria Aparecida Muniz Jorge Dias \& Renata Simões Guimarães e Borges

Tabela 1 - Resultado dos Estilos de Liderança e seus Fatores

\begin{tabular}{lll} 
Estilos de Liderança e Fatores & Média & Desvio Padrão \\
\hline Liderança Transformacional & 7,12 & 3,40 \\
\hline Influência Idealizada & 7,26 & 3,57 \\
\hline Motivação Inspiradora & 7,39 & 3,42 \\
\hline Estímulo Intelectual & 6,88 & 3,62 \\
\hline Consideração Individualizada & 6,97 & 3,63 \\
\hline Liderança Transacional & 6,96 & 2,70 \\
\hline Recompensa Contingente & 6,19 & 3,33 \\
\hline Administração pela Exceção & 7,75 & 2,52 \\
\hline Laissez-faire & 5,85 & 2,46 \\
\hline
\end{tabular}

Nota: $\mathrm{n}=315$. O escore máximo é 12 .

Fonte: Dados da pesquisa

Analisando-se os resultados referentes à variável desempenho de equipes (entregas), conforme Tabela 2, observa-se que os liderados avaliam o desempenho das equipes que pertencem como mediano $(M=17,85, D . P .=3,73)$.

Tabela 2 - Resultado do Desempenho das Equipes

\begin{tabular}{ccc} 
Fatores de Entregas & Média & Desvio Padrão \\
\hline Desempenho das equipes & 17,85 & 3,73 \\
\hline Foco em resultados & 22,08 & 4,97 \\
\hline Foco no cliente & 15,56 & 3,20 \\
\hline Inovação & 18,73 & 4,06 \\
\hline Trabalho em equipe & 21,91 & 5,10 \\
\hline Comprometimento profissional & 10,97 & 2,66 \\
\hline
\end{tabular}

Nota: $\mathrm{n}=315$, o escore máximo é 30 .

Fonte: Dados da pesquisa

Quando se verifica os fatores das entregas, ou seja, aspectos específicos do desempenho, o foco em resultados e o trabalho em equipe foram considerados bons, obtendo as maiores médias $(M=22,08, D . P .=4,97 ; M=21,91, D . P .=5,10$, respectivamente). Por outro lado, o comprometimento profissional é considerado o mais baixo item de entrega pelas equipes com o menor desvio padrão. Ou seja, não há grandes divergências em relação à percepção que os membros das equipes têm um baixo comprometimento profissional $(M=$

$$
\text { 10,97, D.P. = 2,66). }
$$

O resultado da correlação entre os estilos de liderança e a variável dependente desempenho de equipes indica que existe uma correlação positiva entre o estilo transacional e o desempenho $(r=0,567, p<0,001)$. Da mesma forma, os resultados indicam que o estilo de liderança transformacional e o desempenho estão correlacionados $(r=0,535, p<0,001)$. Finalmente, foi verificada uma correlação positiva entre o estilo de liderança Laissez-faire e o desempenho $(r=0,227, p<0,001)$. 
Estilos de liderança e desempenho de equipes no setor público

Para analisar como os estilos de liderança influenciam o desempenho de equipes no governo, foi realizada uma análise de regressão múltipla. Os resultados da análise não confirmam a hipóteses da pesquisa de que o estilo de liderança transformacional seria o principal antecedente do desempenho de equipes. Segundo verifica-se na Tabela 3, o estilo de liderança transacional influencia duas vezes mais o desempenho das equipes do que o estilo de liderança transformacional $(\beta=0,408, p<0,001 ; \beta=0,208, p<0,001$, respectivamente). No entanto, os servidores percebem que o estilo de liderança laissez-faire não influencia o desempenho das equipes $(\beta=0,069, p=0,162)$.

Tabela 3 - Resultado da Análise de Regressão

\begin{tabular}{|c|c|c|c|c|c|}
\hline Coefficients & $\frac{\text { Unstandardized }}{\text { B }}$ & $\begin{array}{c}\text { Coefficients } \\
\text { Std. Error }\end{array}$ & $\begin{array}{c}\begin{array}{c}\text { Standardized } \\
\text { Coefficients }\end{array} \\
\text { Beta }\end{array}$ & $\mathbf{t}$ & Sig. \\
\hline (Constant) & 65,607 & 2,314 & & 28,313 & 0,000 \\
\hline Liderança transacional & 2,681 & 0,657 & 0,408 & 4,083 & 0,000 \\
\hline Liderança transformacional & 1,085 & 0,522 & 0,208 & 2,078 & 0,039 \\
\hline Laissez-faire & & & 0,069 & 1,400 & 0,162 \\
\hline
\end{tabular}

Os resultados sugerem ainda que $35,5 \%$ da variação no desempenho das equipes é explicado pelos estilos de liderança transacional e transformacional $\left(R^{2}\right.$ Adjusted $\left.=0,355\right)$. Isto significa que os estilos de liderança exercem uma forte influência no desempenho das equipes e consequentemente nos resultados organizacionais $[F(1,285)=4,316, p<0,05]$.

\section{CONSIDERAÇÕES FINAIS}

A partir dos resultados obtidos com a pesquisa, pode-se chegar a algumas conclusões relativas aos estilos de liderança presentes nos órgãos e entidades do Poder Executivo de Minas Gerais e suas relações com o desempenho das equipes. Primeiramente, constatou-se que as equipes não percebem tanta diferenciação entre os estilos de liderança transformacional e o transacional, quando avaliam a atuação de seus líderes. No entanto, os servidores responderam que a característica mais forte dos líderes é a administração por exceção que é um traço transacional. Isto que dizer que os líderes procuram e observam desvios das regras e padrões tomando atitudes corretivas necessárias ou intervém apenas quando os padrões esperados não são alcançados. Curiosamente, o segundo e terceiro traços mais observados nos líderes são característicos de uma liderança transformacional. Ou seja, 
são os líderes capazes de comunicar suas altas expectativas, expressam propósitos importantes de maneira simples, além de oferecer uma visão e sentido da missão.

No entanto, ao se analisar a influencia destes estilos nas entregas da equipe, os funcionários entendem que o estilo de liderança transacional é o que faz a equipe obter o melhor desempenho. Ou seja, o líder que esclarece a ligação entre desempenho e recompensas e fornece feedback construtivo para manter os seguidores na tarefa (BASS, 1985; JUNG e AVOBIO, 1999) é o que tende a apresentar equipes de melhor desempenho. Esse resultado contradiz os estudos empíricos de Jung e Avolio (1999), Dionne et al. (2004) e Moynihan et al. (2012) que identificaram o estilo de liderança transformacional como o mais influente no desempenho da equipe.

Algumas implicações surgem com estes resultados. Pode-se inferir que a própria dinâmica das políticas de gestão de pessoas e de gestão por resultados do Poder Executivo de Minas Gerais reforça comportamentos por parte das equipes que demandariam de seus líderes características do estilo de liderança transacional. Nesse sentido, os liderados acabam associando melhor a filosofia das políticas de gestão de pessoas e de gestão por resultados que estabelecem mecanismos que atrelam o desempenho a recompensas. Ou seja, a política de gestão de pessoas, que foca no desenvolvimento, meritocracia e valorização do servidor está pautada em instrumentos que recompensam a formação adicional e resultados satisfatórios. Isto cria uma relação de troca entre Estado-servidor que é estendida para a díade líderliderado refletindo e reforçando as características do líder transacional.

Diante disso, é importante destacar que mensurar desempenho não implica necessariamente na adoção de remuneração variável ou recompensas financeiras. $\mathrm{O}$ desempenho também pode ser mensurado com a finalidade de proporcionar o desenvolvimento das equipes e dos indivíduos, conforme apresentado por Hipólito e Reis (2002). Nos órgãos e entidades do Poder Executivo mineiro, apesar de se trabalhar a perspectiva do desenvolvimento, os resultados da pesquisa levam a concluir que a mensuração do desempenho esta muito mais associada à ideia do servidor ter retornos financeiros, negociando com o líder certas recompensas. Assim, cabe aos responsáveis por estabelecer a política de gestão de pessoas trabalharem outras formas de reconhecimento, que não apenas as recompensas pecuniárias, que contribuam para o desenvolvimento e para a própria geração de resultados.

O estilo de liderança associado ao desempenho para o serviço público é o transacional, cujas características possuem uma interface com o novo modelo da política de gestão de

REAd | Porto Alegre - Edição 80 - º 1 - janeiro/abril 2015 - p. 200-221 
Estilos de liderança e desempenho de equipes no setor público

pessoas, que tem como um de seus pilares a meritocracia. No entanto, é preciso repensar o papel da liderança no setor público uma vez que a liderança transacional excessiva leva a um baixo envolvimento do funcionário. Este aspecto é evidenciado na medida de desempenho que revela que o comprometimento profissional das equipes é o mais baixo índice de desempenho. Pesquisas anteriores mostram que funcionários pouco comprometidos apresentam altos índices de licença médica, baixo desempenho do trabalho, elevada resistência à mudança, e baixos níveis de entrega para o cliente (MEYER et al., 2002).

Portanto, cabe ao governo repensar suas estratégias de gestão de pessoas no sentido de oferecer meios para a liderança transformacional presente na administração pública, conforme apontam os resultados desta pesquisa, melhorar os resultados de desempenho das equipes. Ao invés de focar suas energias no acompanhamento e orientação dos liderados e na distribuição de recompensas (líderes transacionais), o líder deveria assumir um papel inspirador, motivando a equipe e considerando seus membros de forma individualizada, visando atingir as metas e objetivos organizacionais.

Também é importante destacar as baixas médias em relação ao estilo de liderança laissez-faire, ou não liderança. O contexto que os órgãos e entidades do Poder Executivo mineiro vivenciam, de fato, não reforça este estilo de liderança, pois o gestor laissez-faire evita esclarecer suas expectativas e resolver os conflitos, bem como transfere sua autoridade para os liderados abdicando deliberadamente do poder de tomar decisões. Esse resultado é positivo, mas, ao mesmo tempo, ainda exige uma atuação dos responsáveis pela política de gestão de pessoas, para que esse estilo de liderança seja cada vez menor dentre os servidores que ocupam cargo de liderança no Estado de Minas Gerais.

Do ponto de vista acadêmico, o presente trabalho contribuiu para a ampliação dos conhecimentos sobre estilos de liderança na administração pública, apontando contrariamente ao que a literatura coloca sobre a influência do estilo de liderança transformacional. A hegemonia evidenciada para esse estilo de liderança na atualidade não foi reforçada nos resultados obtidos nesta pesquisa, uma vez que na percepção dos servidores, são os líderes transacionais que obtém melhores entregas de suas equipes.

\section{REFERÊNCIAS}

ABRUCIO, F. L. Trajetória Recente da Gestão Pública Brasileira: Um Balanço Crítico e a Renovação da Agenda de Reformas. Rio de Janeiro: RAP Edição Especial Comemorativa, p. 67-86, 2007.

REAd | Porto Alegre - Edição 80 - º 1 - janeiro/abril 2015 - p. 200-221 
Maria Aparecida Muniz Jorge Dias \& Renata Simões Guimarães e Borges

ANDERSON, D. R.; SWEENEY, D. J.; WILLIAMS, T. A. Estatística Aplicada à Administração e Economia. 2.ed. São Paulo: Cengage Learning, 2011.

BABBIE, E. Métodos de Pesquisa de Survey. Belo Horizonte: UFMG, 1999.

BASS, B. M. Leadership and Performance Beyond Expectations. New York: The Free Press, 1985.

BASS, B. M. Bass \& StogdilI's Handbook of Leadership: Theory. Research, and Managerial Applications. New York: The Free Press, 1990.

BASS, B. M.; AVOLIO, B. J. Improving Organizational Effectiveness through Transformational Leadership. Thousand Oaks: Sage Publications, 1994.

BASS, B. M.; AVOLIO, B. J. Full-Range Leadership Development: Manual for the Multifactor Leadership Questionnaire. Palo Alto: Consulting Psychologists Press, 1997.

BASS, B. M.; AVOLIO, B. J. MLQ: Multifactor Questionnaire: Third edition manual and sampler set. Redwood City, CA: Mind Garden, 2000.

BASS, B. M.; AVOLIO, B. J.; JUNG, D. I.; BERSON, Y. Predicting Unit Performance by Assessing Transformational and Transactional Leadership. Journal of Applied Psychology, v. 88, n. 2, p. 207-218, 2003.

BENEDETTI, M. H., HANASHIRO, D. M. M.; POPADIUK, S. Liderança: Uma Relação com Base no Gerenciamento de Stakeholders, A Partir Da Ótica Dos Liderados. Organização \& Sociedade, v.11, n.31, set/dez, 2004.

BERGAMINI, C. W. Liderança: a Administração de Sentido. Revista de Administração de Empresas. São Paulo, v.34, n.3, p.102-114, mai./jun., 1994.

BLAKE, R. R.; MOUTON, J. S. The Managerial Grid. Houston: Gulf Publishing Company, 1964.

BRANNICK, M. T.; PRINCE, C. An Overview of Team Performance Measurement. In:

BRANNICK, M. T.; SALAS E.; PRINCE, C. Team Performance Assessment and

Measurement: Theory, Methods, And Applications. New Jersey: Taylor \& Francis e-Library, 2009.

REAd | Porto Alegre - Edição 80 - N 1 - janeiro/abril 2015 - p. 200-221 
Estilos de liderança e desempenho de equipes no setor público

BURNS, J. M. Leadership. New York: Harper and Row, 1978

COLLIS, J.; HUSSEY, R. Pesquisa em Administração. 2. ed. Porto Alegre: Bookman, 2005.

CRESWELL, J. W. Projeto de Pesquisa: Métodos Qualitativo, Quantitativo e Misto. 2.ed. Porto Alegre: Artmed, 2007.

DE WAAL, A. A. Achieving High Performance in the Public Sector: What Needs to Be Done? Public Performance \& Management Review, v. 34, n. 1, p. 81-103, september, 2010.

DIONNE S. D.; YAMMARINO, F. J.; ATWATER, L. E.; SPANGLER, W. D. Transformacional Leadership and Team Performance. Journal of Organizational Change Management, v. 17, n. 2, p. 177-193, 2004.

DOOREN W. V.; BOUCKAERT, G.; HALLIGAN, J. Performance Management in the Public Sector. New York: Routledge, 2010.

DORFMAN, P. International and Cross-Cultural Leadership. In: PUNNETT, B. J.;

SHENKAR, O. (eds.). Handbook for International Management Research. Cambridge: MA: Blackwell, 1996.

FERLIE, E.; ASHBURNER, L.; FITZGERALD, L.; PETTIGREW, A. The New Public Management in Action. Oxford: Oxford University Press, 1996.

FERNANDES, B. R. Gestão Estratégica de Pessoas com Foco em Competências. Belo Horizonte: Elsevier - Campus, 2013.

GIL, A. C. Métodos e Técnicas de Pesquisa Social. São Paulo: Atlas, 2006.

GUIMARÃES, T. A.; NADER, R. M.; RAMAGEM, S. P. Avaliação de Desempenho de Pessoal: Uma Metodologia Integrada ao Planejamento e Avaliação Organizacionais. Revista de Administração Pública, v. 32, n. 6, p. 43-61, 1998.

HARVEY, D. Condição Pós-Moderna. São Paulo: Loyola, 1999.

HIPÓLITO, J. A.; REIS, G. G. A avaliação como Instrumento de Gestão. In: FLEURY, M. T. L. (Org.). As Pessoas na Organização. São Paulo: Editora Gente, 2002. 
HOLLANDER, E. P. Conformity, Status, and Idiosyncrasy Credit. Psychological Review, v. 65 , p. 117-27, 1958.

HOOD, C. A Public Management for All Seasons? Public Administration, v. 69, p. 3-19, 1991.

JACOBS, T. O.; JAQUES, E. Military Executive Leadership. In: CLARK, K. E.; CLARK, M. B. (Eds). Measures of Leadership. West Orange: Leadership Library of America, 1990.

JONES, D. W.; RUDD, R. D. Transactional, Transformational, or Laissez-Faire Leadership: An Assessment of College of Agriculture Academic Program Leaders (Deans) Leadership Styles. Proceedings of the AAAE Research Conference, v. 34, 2007.

JUNG, D. I.; AVOLIO, B. J. Effects of Leadership Style and Followers' Cultural Orientation on Performance in Group and Individual Task Conditions. Academy of Management Journal, v. 42, n. 2, p.208-218, 1999.

KATZ, D.; KAHN, R. The Social Psychological Of Organizations. 2.ed. New York: Wiley, 1978.

KIRKBRIDE, P. Developing Transformational Leaders: The Full Range Leadership Model in Action. Industrial and Commercial Training, v. 38, n. 1, p. 23-32, 2006.

MARGERISON, C.; MCCANN D.; DAVIES, R. Focus on Team Appraisal. Team Performance Management, v. 1, n. 4 p. 13-18, 1995.

MEYER, J. P.; STANLEY, D. J.; HERSCOVITCH, L.; TOPOLNYTSKY, L. Affective, Continuance, and Normative Commitment to the Organization: A Meta-Analysis of Antecedents, Correlates, and Consequences. Journal of Vocational Behavior, v. 61, n. 1, p. 20-52, 2002.

MOYNIHAN, D. P. The Dynamics of Performance Management: Constructing Information and Reform. Washington: Georgetown Univ. Press, 2008.

MOYNIHAN, D. P.; WRIGHT, B. E.; PANDEY, S. K. Can Transformational Leaders Alter the Experience of Red Tape? International Public Management Journal, v. 15, n. 3, 2012.

MURPHY, K. R. Explaining the Weak Relationship between Job Performance and Rating of Job Performance. Industrial and Organizational Psychology, v. 1, n 2, p. 148-160, 2008. 
Estilos de liderança e desempenho de equipes no setor público

NETO, A. C.; TANURE, B.; SANTOS, C. M. M.; LIMA, G. S. Executivos Brasileiros: na Contramão do Perfil Deificado da Liderança Transformacional. Revista de Ciência da Administração, v. 14, n. 32, p. 35-49, 2012.

NORD, W. R.; FOX, S. The Individual in Organizational Studies: The Great Disappearing Act? In: CLEGG, S.R.; HARDY, C.; NORD, W.R. (eds.). Handbook of Organization Studies, 1996.

ÖZARALLI, N. Effects of Transformational Leadership on Empowerment and Team Effectiveness. Leadership \& Organization Development Journal, v. 24, n. 6, p. 335-344, 2003.

RAUCH, C. F. BEHLING, O. Functionalism: Basis for an Alternate Approach to the Study of Leadership. In: HUNT, J. G.; HOSKING, D. M.; SCHRIESHEIM, C. A.; STEWART, R. (Eds). Leaders and Managers: International Perspectives on Managerial Behaviour and Leadership. Oxford: Pergamon Press, 1984.

ROSS, S. M.; OFFERMANN, L. R. Transformational Leaders: Measurement of Personality Attributes and Work Group Performance. Society for Personality and Social Psychology, v.23, n. 10, p. 1078-1086, 1997.

SADLER, P. Leadership and Organizational Learning. In: DIEKERS, M. et al. Handbook of Organizational Learning and Knowledge. Oxford: Oxford University Press, p. 415-427, 2003.

SHEN, M. J; CHEN, M. C. The Relationship of Leadership, Team Trust and Team Performance: A Comparison of the Service and Manufacturing Industries. Social Behavior and Personality, v.35, n.5, p.643-658, 2007.

SOUZA, V. L.; MATTOS, I. B.; SARDINHA, R. L. L. L.; ALVES, R. C. S. Gestão de Desempenho. Rio de Janeiro: Editora FGV, 2005.

STEWART, J. Transformational Leadership: An Evolving Concept Examined through the Works of Burns, Bass, Avolio, and Leithwood. Canadian Journal of Educational Administration and Policy, v. 54, june, p. 1-29, 2006.

STOGDILL, R. M. Leadership, Membership and Organization. Psychological Bulletin, v. 47, p. $1-14,1950$.

TANNENBAUM, R.; WESCHLER, I. R.; MASSARICK, F. Leadership and Organization. New York: McGraw-Hill, 1961. 
Maria Aparecida Muniz Jorge Dias \& Renata Simões Guimarães e Borges

TICHY, N. M.; COHEN, E. B. The Leadership Engine: How Winning Companies Build Leaders at Every Level. New York: Harper Business, 1997.

TOST; L. P.; GINO, F.; LARRICK, R. P. When Power Makes Others Speechless: The Negative Impact of Leader Power on Team Performance. Academy of Management Journal, v. 56, n. 5, p. 1465-1486, 2013.

WALDMAN, D. A.; RAMIREZ, G. G.; HOUSE, R. J.; PURANAM, P. Does Leadership Matter? CEO Leadership Attributes and Profitability under Conditions of Perceived Environmental Uncertainty. Academy of Management Journal, v. 44, n. 1, p. 134-143, 2001.

WAYNE, S. J.; LIDEN, R. C.; KRAIMER, M. L.; GRAF, I. K. The Role of Human Capital, Motivation and Supervisor Sponsorship in Predicting Career Success. Journal of Organizational Behavior, v. 20, n.5, p. 577-595, september, 1999.

YUKL, G. Managerial Leadership: A Review of Theory and Research. Journal of Management, v. 15, n. 2, p.251-289, 1989.

YUKL, G. Leadership in Organizations. 3 ed. New Jersey: Prentice Hall, 1994. 